\title{
Особенности структурных и оптических свойств InGaN-слоев, полученных методом МПЭ ПА с импульсной подачей потоков металлов
}

\author{
(C) Б.А. Андреев ${ }^{1}$, Д.Н. Лобанов ${ }^{1, \uparrow}$, Л.В. Красильникова ${ }^{1}$, К.Е. Кудрявцев ${ }^{1}$, А.В. Новиков ${ }^{1}$, \\ П.А. Юнин ${ }^{1}$, М.А. Калинников ${ }^{1}$, Е.В. Скороходов ${ }^{1}$, М.В. Шалеев ${ }^{1}$, З.Ф. Красильник ${ }^{1,2}$ \\ ${ }^{1}$ Институт ффизики микроструктур Российской академии наук, \\ 603087 Нижний Новгород, Нижегородская обл., Россия \\ ${ }^{2}$ Нижегородский государственный университет им. Н.И. Лобачевского, \\ 603950 Нижний Новгород, Россия \\ ฯ E-mail: dima@ipmras.ru
}

Поступила в Редакцию 12 апреля 2021 г.

В окончательной редакции 19 апреля 2021 г.

Принята к публикации 19 апреля 2021 г.

\begin{abstract}
Представлены результаты исследований свойств слоев InGaN с высоким содержанием InN (80-90\%), полученных методом молекулярно-пучковой эпитаксии с плазменной активацией азота на сапфировых подложках с буферными слоями AlN/GaN. Формирование InGaN-слоев производилось методом модулирования потоков металлов (MME - metal modulated epitaxy), а также в азот- и металлобогащенных условиях. Обнаружено, что применение метода ММЕ приводит к снижению плотности прорастающих дислокаций в слоях InGaN. Тем не менее, несмотря на большую плотность дислокаций, наименьший порог стимулированного излучения $\sim 20$ кВт/см² при $77 \mathrm{~K}$ был получен для слоя $\operatorname{In}_{0.8} \mathrm{Ga}_{0.2} \mathrm{~N}$, выращенного в азотобогащенных условиях, что связывается с наименьшей фоновой концентрацией электронов в этом образце $\left(1.6 \cdot 10^{19} \mathrm{~cm}^{-3}\right)$.
\end{abstract}

Ключевые слова: нитрид индия и галлия, молекулярно-пучковая эпитаксия, дислокации, фотолюминесценция, стимулированное излучение.

DOI: 10.21883/FTP.2021.09.51292.22

\section{1. Введение}

Нитриды металлов III группы (AlN, GaN и InN) и их сплавы в настоящее время являются одними из наиболее используемых материалов для создания различных оптоэлектронных приборов. Эти прямозонные полупроводники охватывают широкий диапазон длин волн от ультрафиолетового (УФ) до инфракрасного (ИК) [1]. Тем не менее на текущий момент коммерчески освоенным является довольно узкий диапазон от ближнего УФ (> $300 \mathrm{нм})$ до видимого диапазона с длинами волн $\sim 500$ нм $[2,3]$. Продвижение в область больших длин волн сопровождается существенным падением внутренней и внешней квантовых эффективностей излучения, что в совокупности с тем, что AlInGaPисточники становятся неэффективными на длинах волн короче красного света, формирует так называемую „зеленую“ щель $[3,4]$. В красной области спектра лазерная генерация получена лишь одной научной группой на основе планарных структур с квантовыми точками InGaN на крайне дорогих подложках $\mathrm{GaN}$, что затрудняет их практическое применение [5]. Одним из путей продвижения в ИК область спектра является формирование одномерных InGaN-нитей с InN-квантовыми ямами в них, на которых недавно продемонстрирована лазерная генерация на длинах волн до 1.33 мкм [6]. Однако существенными недостатками данного типа структур по сравнению с планарными структурами являются сложность формирования лазерных структур и низкая выходная мощность излучения.

В то же время авторами настоящей работы было продемонстрировано стимулированное излучение в диапазоне длин волн 1.6-1.9 мкм из планарных слоев $\mathrm{InN}$, полученных методом молекулярно-пучковой эпитаксии с плазменной активацией азота (МПЭ ПА) на сапфировых подложках с буферными слоями GaN/AlN [7]. Было показано, что параметры стимулированного излучения (длина волны и пороговая мощность) существенным образом зависят от структурного качества $\mathrm{InN}$-слоев, шероховатости их поверхности и фоновой концентрации электронов [7].

Формирование высококачественных слоев InGaN c высоким содержанием In, необходимое для создания источников излучения в практически важном диапазоне длин волн 1.3-1.55 мкм, связано с существенными технологическими трудностями. Основными проблемами здесь являются термическое разложение [8], спинодальный распад [9] и сегрегация In [10]. Одним из основных способов избежать вышеозначенных проблем является, независимо от конкретного метода эпитаксии, снижение температуры роста (см., например, работу [11]). Для получения методом МПЭ ПА однородных InGaN-слоев с содержанием $[\mathrm{In}]>45 \%$ температуру роста необходимо снижать до $\leq 500^{\circ} \mathrm{C}$. Даже незначительное превышение температуры роста выше этого значения приводит к спинодальному распаду растущего слоя, а затем, при 
$T \sim 545^{\circ} \mathrm{C}$, и к его термическому разложению [12]. Столь низкие температуры роста ограничивают, как и в случае InN, применение газофазных методов эпитаксии и ведут к снижению кристаллического качества выращиваемых слоев InGaN [13-15].

Как было показано ранее в работе [7], большая шероховатость поверхности слоев InN может негативно сказываться на возможности достижения в них стимулированного излучения. Известно, что для формирования методом МПЭ ПА слоев нитридов металлов III группы с низкой шероховатостью поверхности предпочтительны металлобогащенные условия роста [16]. Однако в случае роста соединений InGaN в таких условиях на поверхности происходит накопление металлического In, избавиться от которого довольно затруднительно. Кроме этого, ранее при исследовании структурных и оптических свойств слоев InN было показано, что рост в металлобогащенных условиях приводит к формированию в них глубоких акцепторных центров, включений металлической фазы и, как следствие, росту порога и отсутствию стимулированного излучения [17].

В формировании высококачественных слоев InGaN c высоким содержанием In может помочь метод модулирования потоков металлов (MME - metal modulated epitaxy) с периодическим ростом в металлобогащенных условиях, и последующей выдержкой поверхности роста под потоком азота для связывания накопившегося металла. Этот метод продемонстрировал свою эффективность для повышения кристаллического качества бинарных соединений $\mathrm{A}^{\mathrm{III}} \mathrm{N}$ [18-20]. В работе [21] подобным образом были получены слои $\mathrm{InGaN} \mathrm{c} \mathrm{долей} \mathrm{индия}$ $22 \%<[\operatorname{In}]<72 \%$ хорошего структурного качества: с шириной пика рентгеновского отражения $(0002)<416$ угл.сек и шероховатостью поверхности $<1$ нм. Для получения высококачественных слоев $\mathrm{InGaN}$ в режиме MME важно точно контролировать количество металла на поверхности, так как накопление избыточного металла может привести, кроме латеральной неоднородности состава, к сегрегации индия и периодической модуляции состава в направлении роста. Как утверждают авторы работ [21,22], рост в металлобогащенных условиях не обязательно должен приводить к сегрегации металлического In на поверхности, так как проявление этого эффекта зависит от количества накопленного металла на поверхности. В случае если количество избыточного металла на поверхности не превышает одного бислоя, сегрегации In не происходит и возможно получение однородных по составу слоев InGaN в режиме MME роста. В случае накопления на поверхности более одного бислоя сегрегация In в режиме MME роста приводит к формированию периодической структуры с чередованием слоев InGaN с разным содержанием In [22].

Приведенные результаты показывают, что режим роста ММЕ успешно использовался для получения планарных InGaN-слоев с долей [In] до 70\% [21,22]. В то же время для создания источников излучения на диапазон длин волн $\geq 1.3$ мкм необходимы слои $\mathrm{InGaN}$ с большей долей In.
Цель данной работы - определение влияния ростовых условий метода MME на возможность реализации стимулированного излучения. Представлены результаты исследования процесса роста слоев InGaN с содержанием In от 80 до 90\% при различных режимах (в том числе, методом ММЕ). Изучено влияние количества накопленного за время металлобогащенного роста металла на поверхности в одном цикле (от 0.5 до $2 \mathrm{MC}$ ) на структурные и оптические свойства слоев InGaN. Приводятся результаты сравнения свойств этих слоев со слоями, выращенными в металл- и азотобогащенных условиях.

\section{2. Методика эксперимента}

Рост слоев InGaN проводился на подложках $c-\mathrm{Al}_{2} \mathrm{O}_{3}$ методом МПЭ ПА на установке STE $3 \mathrm{~N} 3$ (3АO „HTO“). На сапфировых подложках диаметром $2^{\prime \prime}$ со слоем Ті толщиной 400 нм, нанесенным на обратную сторону, последовательно выращивались высокотемпературные буферные слои AlN (200 нм) и GaN (700 нм) при температурах 820 и $710^{\circ} \mathrm{C}$ соответственно. На таких буферных слоях $\mathrm{GaN} / \mathrm{AlN}$ осуществлялся рост слоя InGaN с содержанием In 80-90\%. Рост слоев InGaN проходил при температурах $450-460^{\circ} \mathrm{C}$. В процессе роста слоев InGaN методом MME потоки Ga и In не менялись и по данным калибровок составляли для $\mathrm{Ga} \sim 0.06$ мкм/ч и для In $\sim 0.27$ мкм/ч. Поток азота $(2.5 \mathrm{sccm}$, стандартных кубических сантиметра в минуту) и мощность разряда плазменного источника азота RF Atom Source HD25 $(130 \mathrm{BT})$ также оставались неизменными в процессе роста слоев InGaN методом MME, что давало скорость осаждения активного азота $\sim 0.28$ мкм/ч для слоя $\mathrm{InGaN}$ с содержанием $[\mathrm{In}]=80-90 \%$.

Для сравнения были выращены также слои InGaN в азот- и металлобогащенных условиях, без прерывания потоков металлов. Азотобогащенные условия достигались увеличением мощности разряда плазменного источника азота до $160 \mathrm{~B}$, что соответствовало потоку активного азота $\sim 0.39$ мкм/ч и соотношению потоков элементов III и V групп $\sim 0.85$. Рост в металлобогащенных условиях проходил при соотношении потоков III/V 1.1. Толщина InGaN, полученных методом MME, составляла 330-370 нм, а выращенных в азот- и металлобогащенных условиях 600-650 нм.

Выращенные образцы исследовались методами атомно-силовой микроскопии (АСM), рентгенодифракционного анализа (РД), фотолюминесценции (ФЛ) и эффекта Холла. Спектры ФЛ регистрировались с поверхности образца при оптической накачке непрерывным лазером (длина волны возбуждения $\lambda_{\mathrm{ex}}=0.8$ мкм, мощность до 500 мВт). Режим стимулированной эмиссии реализовывался при возбуждении импульсным оптическим параметрическим генератором на длине волны $\lambda=0.65$ мкм, с энергией до 3 мДж в импульсе длительностью 10 нс при частоте их повторения 10 Гц. Пятно 


\begin{tabular}{|c|c|c|c|c|c|}
\hline Режим роста InGaN & $\begin{array}{l}\text { Азотобогащенный } \\
\text { (N-rich) III/V 0.85 }\end{array}$ & $\begin{array}{l}\mathrm{MME} \\
0.5 \mathrm{MC}\end{array}$ & $\begin{array}{l}\text { MME } \\
1 \mathrm{MC}\end{array}$ & $\begin{array}{l}\text { MME } \\
2 \mathrm{MC}\end{array}$ & $\begin{array}{l}\text { Металлобогащенный } \\
\text { (Me-rich) III/V } 1.1\end{array}$ \\
\hline Винтовые дислокации $\left(\mathrm{cm}^{-2}\right)$ & $2.2 \cdot 10^{9}$ & $2 \cdot 10^{9}$ & $1.9 \cdot 10^{9}$ & $1.5 \cdot 10^{9}$ & $1.9 \cdot 10^{9}$ \\
\hline Краевые дислокации $\left(\mathrm{cm}^{-2}\right)$ & $5.9 \cdot 10^{10}$ & $4.7 \cdot 10^{10}$ & $3.8 \cdot 10^{10}$ & $4.1 \cdot 10^{10}$ & $1.2 \cdot 10^{11}$ \\
\hline Среднеквадратичная шероховатость (нм) & 4 & 5 & 5 & 5 & $<1$ \\
\hline Содержание индия (\%) & 80 & 81 & 82 & 81 & 90 \\
\hline Концентрация электронов по методу Холла $\left(\mathrm{cm}^{-3}\right)$ & $1.6 \cdot 10^{19}$ & $3.1 \cdot 10^{19}$ & $2.1 \cdot 10^{19}$ & $2.7 \cdot 10^{19}$ & $2.3 \cdot 10^{19}$ \\
\hline Подвижность электронов по методу Холла $\mathrm{cm}^{2} /(\mathrm{B} \cdot \mathrm{c})$ & 630 & 364 & 447 & 457 & 417 \\
\hline Положение пика ФЛ при 77 К (эВ) & 1.047 & 1.108 & 1.033 & 1.072 & 1.007 \\
\hline Положение пика СИ при 77 К (эВ) & 1.012 & 1.057 & 0.978 & 1.032 & нет СИ \\
\hline Порог СИ при $77 \mathrm{~K}$ (кВт/см²) & 20 & 200 & 60 & 130 & нет СИ \\
\hline
\end{tabular}

накачки фокусировалось на поверхности образца в полоску шириной $\sim 200$ мкм и длиной до 10 мм, излучение собиралось от края образца и регистрировалось с помощью монохроматора Acton 2300i и многоэлементного диодного детектора OMA-V, диапазон работы которого составлял $\lambda=0.8-2.1$ мкм.

\section{3. Результаты и их обсуждение}

Схема роста, открытия и закрытия заслонок в режиме MME представлена на рис. 1. При неизменных потоках материалов накопление разного количества металла на поверхности в процессе ММЕ роста осуществлялось за счет разного времени роста в металлобогащенных условиях $\left(t_{1}\right)$ (рис. 1). Превышение суммарного потока металлов над потоком активного азота составляло $\sim 0.05$ мкм/ч, что для образцов с $t_{1}=10,20$ и $30 \mathrm{c}$ приводило к накоплению на поверхности роста $0.5,1$ и $2 \mathrm{MC}$ металлов (In и $\mathrm{Ga}$ ) соответственно. Последующая выдержка поверхности роста под потоком азота при закрытых потоках металлов $\left(t_{2}\right)$ составляла $t_{2}=10$ с для $t_{1}=10$ с и $t_{1}=20$ с и $t_{2}=15$ с для $t_{1}=30 \mathrm{c}$.

Связывание металла в процессе выдержки структуры под потоком азота контролировалось по смене картины

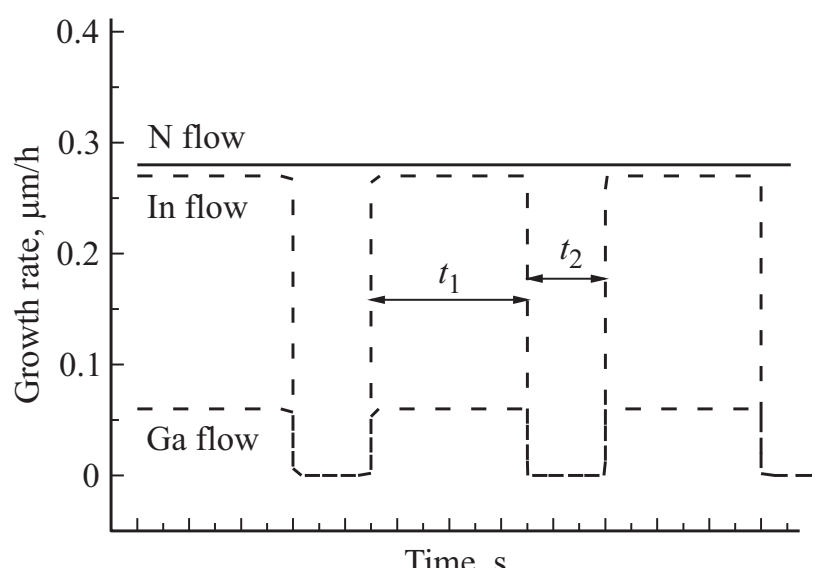

Рис. 1. Зависимость потоков от времени в процессе ММЕ роста InGaN-слоя. дифракции быстрых электронов на отражение (ДБЭО). Слабо выраженные полосовые рефлексы на карте ДБЭО после роста в металлобогащеных условиях менялись на яркую, хорошо известную для InGaN [0001] картину реконструкции $(\sqrt{3} \times \sqrt{3})$ [23]. Несмотря на существенную разницу в количестве накопленного на поверхности роста металла, все образцы, выращенные в режиме $\mathrm{MME}$, по данным АСМ измерений, продемонстрировали примерно одинаковую морфологию поверхности со среднеквадратичной шероховатостью поверхности $\sim 6$ нм (рис. 2). Даже в случае накопления бислоя металла на поверхности $\left(t_{1}=30 \mathrm{c}\right)$ выглаживания поверхности роста не происходит. При этом шероховатость поверхности InGaN-слоев, выращенных в режиме MME, оказалась даже несколько больше по сравнению с ростом в азотобогащенных условиях (III/V $\sim 0.85)$, где она составляла $\sim 4$ нм. Кардинальное снижение шероховатости поверхности до 1 нм происходило при переходе к металлобогащенным условиям роста (III/V 1.1) (рис. $2, c)$. Возможной причиной высокой шероховатости поверхности InGaN слоев, полученных методом MME, по сравнению с работами других авторов является недостаточное количество металла на поверхности роста. Так, при формировании методом MME слоев $\mathrm{GaN}$ было показано [18], что для снижения шероховатости необходимо накапливать на поверхности $>2 \mathrm{MC}$ в фазе ее роста. Как будет показано далее, при формировании InGaN-слоев с высоким содержанием In такой режим MME роста приводит к сегрегации In и невозможности получения однородных по составу слоев. Низкая шероховатость слоев InGaN, полученных в работе [22], может быть обусловлена их малой толщиной $(50-60 \mathrm{Hм})$ и более низкими температурами роста $\left(400-450^{\circ} \mathrm{C}\right)$ по сравнению с температурами, используемыми в настоящей работе.

Исследования кристаллического качества InGaN слоев, проведенные методами рентгенодифракционного анализа, показали, что все слои In GaN являются полностью релаксированными. Содержание [In] в слоях InGaN, полученных методом ММЕ и в азотобогащенных условиях, составляло $\sim 80 \%$, в металлобогащенных условиях $\sim 90 \%$ (см. таблицу). Было обнаружено, что, несмотря на меньшую толщину, слои, выращенные методом 

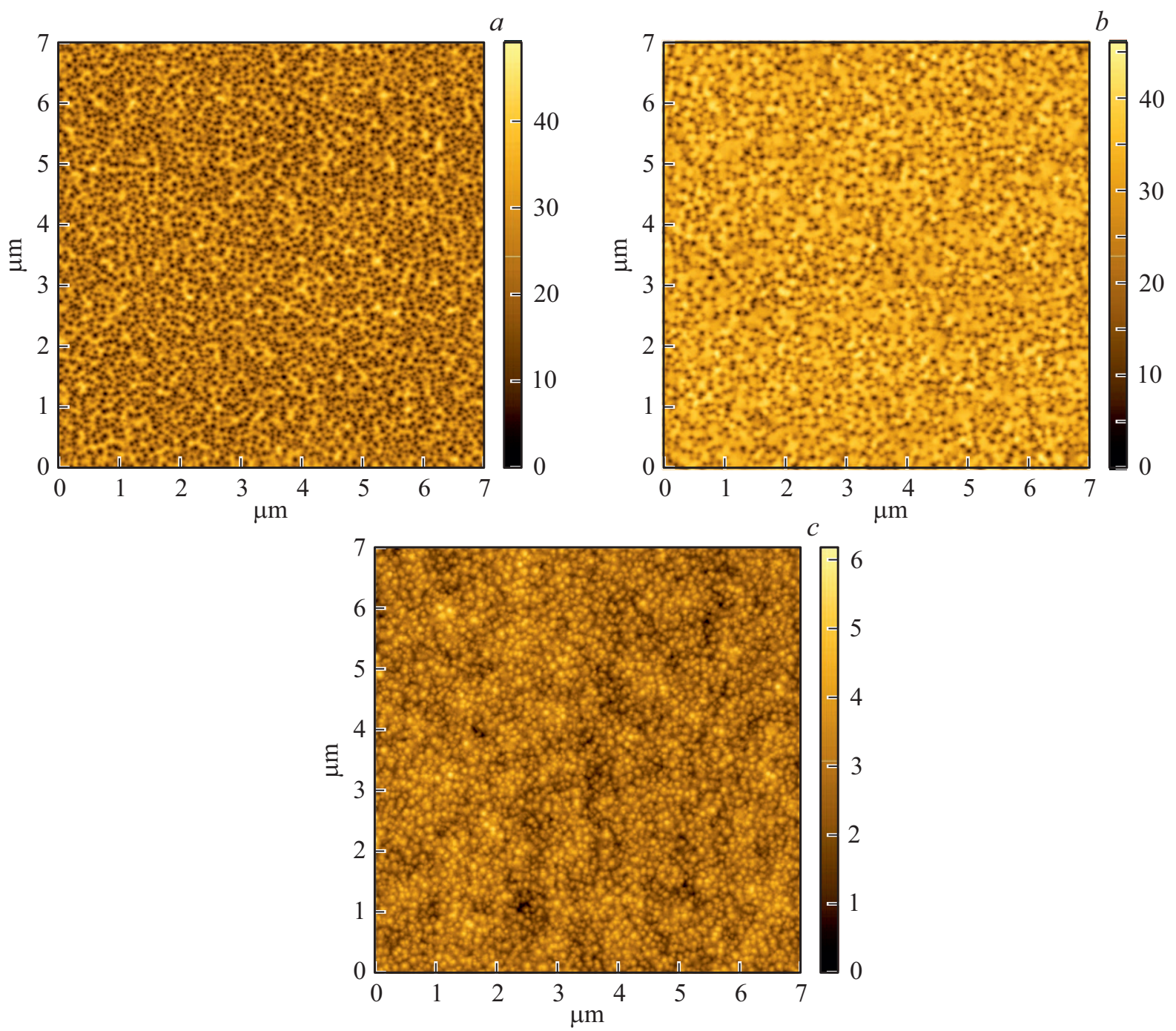

Рис. 2. АСМ-снимки образцов с InGaN-слоями, выращенными методом ММE с накопленным металлом на поверхности $0.5(a)$ и $2 \mathrm{MC}(b)$ и АCM-снимок образца с InGaN-слоем, выращенным в металлобогащенных условиях III/V $1.1(c)$.

MME, характеризуются меньшей плотностью прорастающих дислокаций, чем слои, полученные в азотобогащенных условиях (см. таблицу). Снижение плотности прорастающих дефектов наблюдается с увеличением количества накопленного на поверхности металла до 1 МС. Эффективность метода ММЕ для снижения плотности прорастающих дислокаций при росте нитридов металлов III группы на рассогласованных подложках продемонстрирована в работах разных групп [24,25]. При увеличении накопленного металла до $2 \mathrm{MC}$ наблюдается рост плотности прорастающих дислокаций (см. таблицу). Похожее поведение плотности дислокаций от параметров MME роста наблюдали и авторы работы [18]. Переход к металлобогащенным условиям роста приводит к значительному ( 2 раз) увеличению плотности дислокаций в InGaN-слое, даже по сравнению с ростом в азотобогащенных условиях (см. таблицу). Возможной причиной этого является развитая трехмерная морфология поверхности InGaN-слоев, вы- ращенных в азотобогащенных условиях, благодаря которой отклонение от распространения прорастающих дислокаций вдоль направления роста с последующим их взаимодействием и аннигиляцией происходит более эффективно [26,27].

Еще одной особенностью $\mathrm{InGaN}$-слоя, полученного методом MME с накоплением металла до $2 \mathrm{MC}$, является обнаруженный при $\omega-2 \theta$ сканировании сигнал от короткопериодной решетки (рис. 3), период которой $(2.7-2.9$ нм $)$ соответствует количеству материала, осажденного за один цикл ММЕ роста. Этот результат говорит о начавшейся сегрегации In, формировании периодических InGaN-слоев разного состава, и противоречит результату работы [22], где говорилось об отсутствии сегрегации In при росте InGaN-слоев методом MME, если количество металла на поверхности не превышает 2 МС. Возможной причиной этого является то, что при наличии на поверхности до $\leq 1 \mathrm{MC}$ атомов In и Ga расслоения (сегрегации) In не возникает - места на 


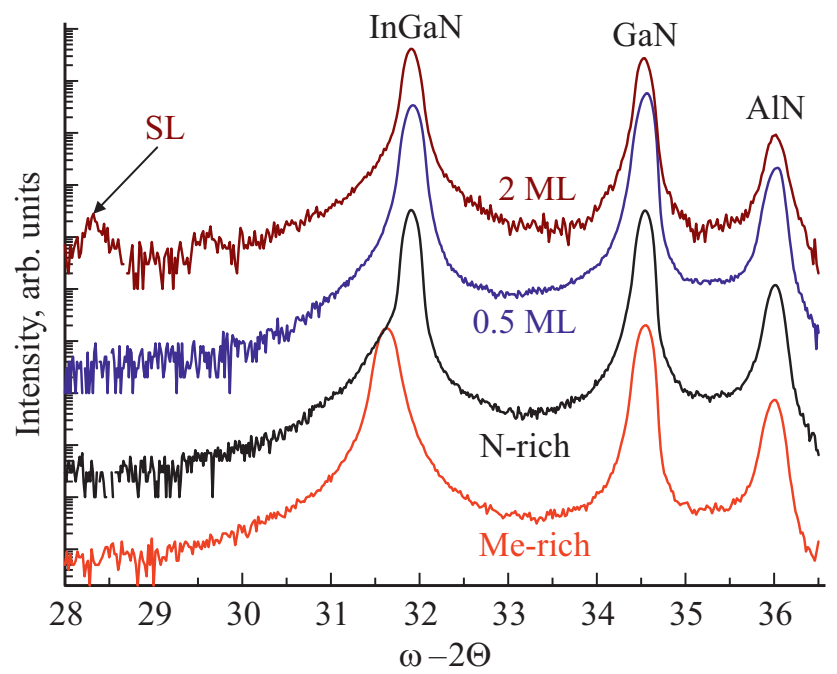

Рис. 3. (0002) $\omega-2 \theta$ спектры рентгеновской дифракции образцов с InGaN-слоями, выращенными в различных условиях (указаны под соответствующим спектром). Пик, обозначенный SL, указывает на появление сверхрешетки $\mathrm{In}_{x} \mathrm{Ga}_{1-x} \mathrm{~N} / \mathrm{In}_{y} \mathrm{Ga}_{1-y} \mathrm{~N}$ с периодическими слоями InGaN разного состава в образце с 2 MC металла, накопленного на поверхности. (Цветной вариант рисунка представлен в электронной версии статьи).

поверхности роста хватает всем атомам металла. Когда появляется второй МС атомов металлов, то между нижним и верхним слоями возникает неравнозначный обмен атомами, приводящий к выталкиванию (сегрегации) атомов In на поверхность, в верхний МС. В работе [22] такой сегрегации атомов In не наблюдали, возможно, из-за более низких температур роста, препятствующих обмену атомами между двумя слоями атомов металлов.

Измерения методом Холла показали, что среди исследованных структур наименьшую фоновую концентрацию электронов $\sim 1.6 \cdot 10^{19} \mathrm{~cm}^{-3}$ имеет слой InGaN, выращенный в азотобогащенных условиях (см. таблицу). Слои InGaN, полученные методом MME или выращенные в металлобогащенных условиях, демонстрируют близкие фоновые концентрации электронов $(2.3-3.1) \cdot 10^{19} \mathrm{~cm}^{-3}$. Подвижность электронов падает в $\sim 2$ раза при переходе от азотобогащенных условий к росту методом MME, несмотря на уменьшение плотности прорастающих дислокаций, что может быть связано с увеличением концентрации электрически активных дефектов (не относящихся к дислокациям) и фоновой концентрации электронов. При переходе к металлобогащенным условиям роста InGaN подвижность электронов также снижается, что в этом случае связывается с более высокой плотностью дислокаций в этом образце (см. таблицу).

Высокая фоновая концентрация электронов в слоях InGaN может быть обусловлена разными факторами. В работах [28-30] было показано, что дислокации могут формировать мелкие донорные центры за счет оборван- ных связей. Еще одной причиной может быть неконтролируемое встраивание примеси в растущий слой [31]. В частности, такие остаточные примеси как углерод и кислород могут формировать донорные центры и выступать основной причиной высокой фоновой концентрации электронов в слоях InN, выращенных при низких температурах, что было показано в работе [7].

В слоях, полученных методом MME, отличие в концентрациях электронов коррелирует с различием в плотности прорастающих дислокаций, и наименьшей фоновой концентрацией электронов обладает образец MME $1 \mathrm{ML}$ с наименьшей плотностью прорастающих дислокаций. В слоях InGaN, выращенных в азот- и металлобогащенных условиях, плотность дислокаций выше, чем в ММЕ образцах, однако фоновая концентрация электронов ниже. Предполагается, что это может быть связано с более высокой средней скоростью роста последних образцов, а следовательно, с меньшей вероятностью встраивания в них фоновых примесей.

Описание экспериментальных спектров ФЛ (рис. 4) в рамках модели межзонных переходов [32] позволило определить эффективную ширину запрещенной зоны $E_{g}$ и положение уровня Ферми для исследованных структур. Содержание $\operatorname{In}(x)$ в слоях $\operatorname{In}_{x} \mathrm{Ga}_{1-x} \mathrm{~N}$, определенное с помощью эмпирической зависимости [32] $E_{g}(n, T=77 \mathrm{~K})=3.493-2.843 \cdot x$ $-2.5 \cdot x(1-x)(э \mathrm{~B})$, хорошо согласуется с результатами определения состава методом рентгенодифракционного анализа. На положение сигнала ФЛ слоев $\mathrm{InGaN}$ кроме состава влияет ряд факторов, таких как флуктуации зонного потенциала, вызванные, в частности, неоднородностью состава, различная фоновая концентрация электронов, приводящая к смещению уровня Ферми в образцах, и другие. В исследованных InGaN-слоях с близким содержанием In синяя граница и положение максимума ФЛ отслеживают фоновую концентрацию электронов в образце (см. таблицу и рис. 4), сдвигаясь к высоким

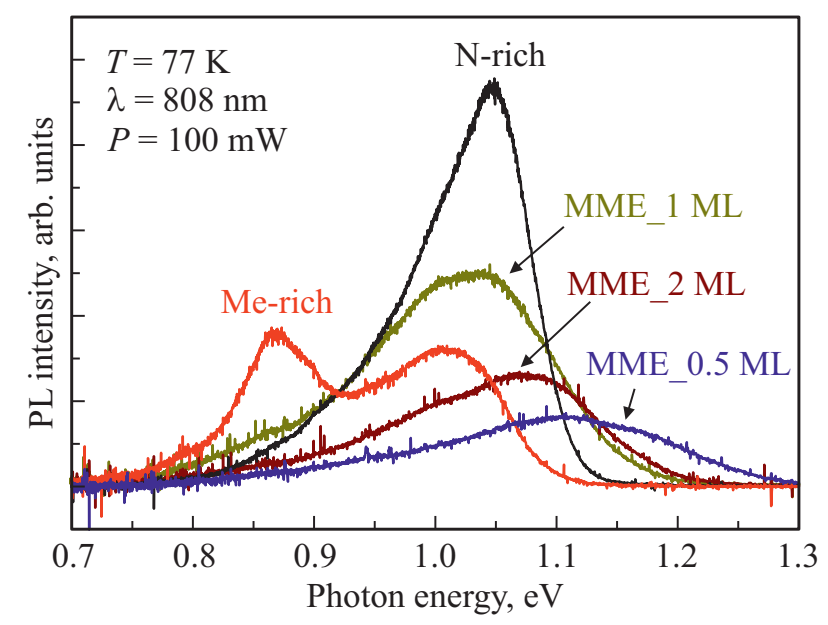

Рис. 4. Спектры спонтанной фотолюминесценции образцов с $\mathrm{InGaN-cлоями,} \mathrm{выращенными} \mathrm{в} \mathrm{различных} \mathrm{условиях.} T=77 \mathrm{~K}$. 


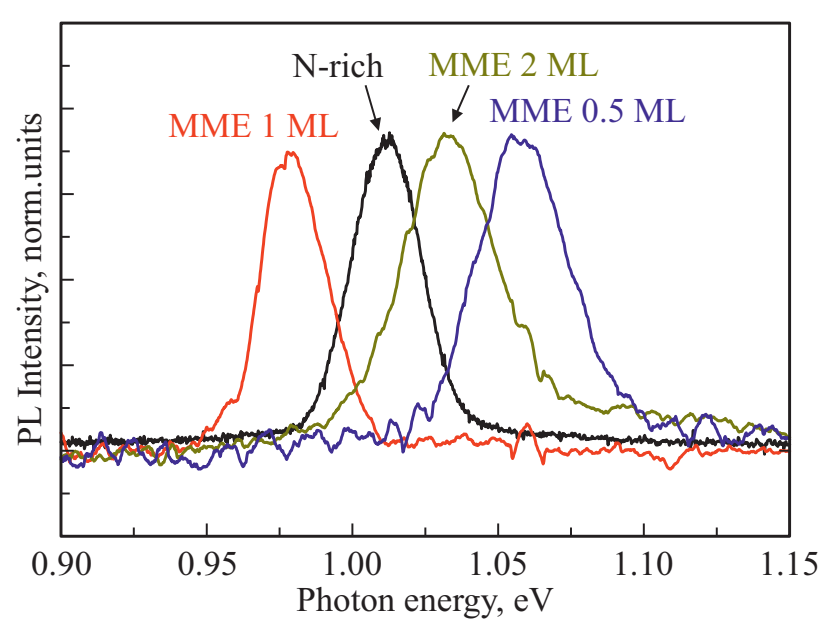

Рис. 5. Нормированные спектры стимулированной эмиссии образцов с InGaN-слоями, полученных в различных условиях. $T=77 \mathrm{~K}$.

энергиям с ростом концентрации свободных электронов, в соответствии с эффектом Бурштейна-Мосса.

В спектре образца, выращенного в металлобогащенных условиях, хорошо различимы два пика, разнесенных по энергии на $\sim 135$ мэВ (рис. 4). Более высокоэнергетичный пик связывается, как и в случае рассмотренных выше сигналов ФЛ от InGaN-слоев, выращенных в азотобогащенных условиях и в режиме MME, с межзонными переходами, а также с переходами из зоны проводимости в хвосты состояний валентной зоны [33]. Низкоэнергетический пик ФЛ вызван переходами из зоны проводимости в акцепторные состояния, связанные с избыточными атомами индия, вследствие металлобогащенных условий роста [17].

От всех образцов, кроме структуры со слоем InGaN, полученным в металлобогащенных условиях, было получено стимулированное излучение (СИ) при импульсной оптической накачке (рис. 5). Отсутствие СИ от InGaNслоя, полученного в металлобогащенных условиях, связывается с формированием глубоких акцепторных центров, вызванных избыточным In [17]. Положение сигнала СИ коррелирует с положением максимума сигнала ФЛ соответствующего образца и сдвинуто относительно него в сторону меньших энергий на 35-50 мэВ (см. таблицу).

Наименьший порог в $\sim 20$ кВт/см ${ }^{2}$ при $77 \mathrm{~K}$ был получен для InGaN-образца, выращенного в азотобогащенных условиях, что существенно ниже порога в $\sim 60 \mathrm{BT} / \mathrm{cm}^{2}$, полученного для InN-слоя с близкой плотностью прорастающих дислокаций и фоновой концентрацией электронов [7]. Одной из причин этого может быть увеличение вероятности излучательной рекомбинации за счет увеличения плотности состояний в валентной зоне и зоне проводимости, так как с увеличением содержания $\mathrm{Ga}$ в InGaN-слоях происходит увеличение эффективных масс носителей зарядов. Также снижение порога СИ может быть связано с уменьшением коэффициента ожерекомбинации в InGaN по сравнению с InN [34]. Как было показано в работе [7], именно фоновая концентрация электронов и оже-рекомбинация определяют порог стимулированного излучения при низких температурах в InN. Образцы с InGaN-слоями, полученными методом MME, также продемонстрировали СИ, однако пороги СИ для них, несмотря на меньшую плотность дислокаций, оказались выше, что связывается с большей фоновой концентрацией электронов в них (см. таблицу). Наименьший порог стимулированного излучения среди слоев InGaN, полученных методом MME, в $~ 60$ кВт/см ${ }^{2}$ наблюдался для образца MME $1 \mathrm{ML}$, что связывается с наименьшей плотностью прорастающих дислокаций и наименьшей фоновой концентрацией электронов (см. таблицу). Сопоставление параметров InGaN-слоев, полученных в различных условиях, с порогом достижения СИ излучения в них показывает, что в исследованных структурах с фоновой концентрацией электронов $>10^{19} \mathrm{~cm}^{-3}$ именно этот параметр оказывает основное влияние на их излучательные свойства.

\section{4. Заключение}

Продемонстрировано, что формирование слоев InGaN с долей $[\mathrm{In}] \sim 80 \%$ методом ММЕ с импульсной подачей потоков металлов III группы приводит к снижению плотности прорастающих дислокаций по сравнению с непрерывной подачей потоков в азот- и металлобогащенных условиях. При росте слоев InGaN методом MME при температурах $450-460^{\circ} \mathrm{C}$ было обнаружено, что при накоплении в одном цикле на поверхности роста более $1 \mathrm{MC}$ металлов наблюдается формирование периодической структуры в направлении роста из слоев In GaN различного состава, вызванное сегрегацией In. Впервые в ИК диапазоне длин волн от планарных InGaN-слоев с долей $[\mathrm{In}] \sim 80 \%$ наблюдалось стимулированное излучение при низких температурах. Наименьший порог при температуре $77 \mathrm{~K}$ был получен для слоя $\operatorname{In}_{0.8} \mathrm{Ga}_{0.2} \mathrm{~N}$, выращенного в азотобогащенных условиях, и составил $\sim 20 \mathrm{\kappa} \mathrm{BT} / \mathrm{cm}^{2}$. Несмотря на лучшее структурное совершенство, порог СИ в образцах с In $\mathrm{GaN}$-слоями, полученными методом MME, оказался существенно выше, что связывается с большей фоновой концентрацией электронов в них. Наименьший порог для них составил $\sim 60$ кВт/см ${ }^{2}$ при $77 \mathrm{~K}$ для образца с накопленным за один цикл $1 \mathrm{MC}$ металла, обладающим наименьшей плотностью дислокаций и наименьшей фоновой концентрацией электронов среди ММЕ образцов.

\section{Финансирование работы}

Работа выполнена в рамках государственного задания Министерства науки и высшего образования Российской Федерации (тема № 0030-2021-0019). 


\section{Конфликт интересов}

Авторы заявляют, что у них нет конфликта интересов.

\section{Список литературы}

[1] C.J. Humphreys. MRS Bull., 33, 459 (2008).

[2] Q. Guo, R. Kirste, S. Mita, J. Tweedie, P. Reddy, B. Moody, Y. Guan, S. Washiyama, A. Klump, Z. Sitar, R. Collazo. J. Appl. Phys., 126, 223101 (2019).

[3] Q. Zhou, M. Xu, H. Wang. Opto-Electron. Rev., 24 (1), 1 (2016).

[4] K.A. Bulashevich, A.V. Kulik, S.Y. Karpov. Phys. Status Solidi A, 212, 914 (2015).

[5] T. Frost, A. Banerjee, Kai Sun, S.L. Chuang, P. Bhattacharya. IEEE Quant. Electron., 49 (11), 923 (2013).

[6] P. Bhattacharya, A. Hazari, S. Jahangir. Proc. SPIE, 10553, 1055302 (2018).

doi: $10.1117 / 12.2302548$

[7] B.A. Andreev, K.E. Kudryavtsev, A.N. Yablonskiy1, D.N. Lobanov, P.A. Bushuykin, L.V. Krasilnikova, E.V. Skorokhodov, P.A. Yunin, A.V. Novikov, V.Yu. Davydov, Z.F. Krasilnik. Sci. Rep., 8, 9454 (2018). doi:10.1038/s41598-018-27911-2

[8] C. Adelmann, R. Langer, G. Feuillet, B. Daudin. Appl. Phys. Lett., 75, 3518 (1999).

[9] G.B. Stringfellow. J. Cryst. Growth, 312, 735 (2010).

[10] H. Chen, R.M. Feenstra, J.E. Northrup, T. Zywrzet. J. Vac. Sci. Technol. B, 18, 2284 (2000).

[11] M. Moseley, B. Gunning, J. Greenlee, J. Lowder, G. Namkoong, W.A. Doolittle. J. Appl. Phys., 112, 014909 (2012).

[12] H. Komaki, T. Nakamura, R. Katayama, K. Onabe, M. Ozeki, T. Ikari. J. Cryst. Growth, 301, 473 (2007).

[13] F.K. Yam, Z. Hassan. Superlat. Microstruct., 43 (1), 1 (2008).

[14] C.A. Chang, C.F. Shih, N.C. Chen, T.Y. Lin, K.-Sh. Liu. Appl. Phys. Lett., 85 (25), 6131 (2004).

[15] B.N. Pantha, J. Li, J.Y. Lin, H.X. Jiang. Appl. Phys. Lett., 93, 182107 (2008).

[16] E. Dimakis, E. Iliopoulos, K. Tsagaraki, A. Georgakilas. Appl. Phys. Lett., 86, 133104 (2005). doi: $10.1063 / 1.1891292$

[17] Б.А. Андреев, Д.Н. Лобанов, Л.В. Красильникова, П.А. Бушуйкин, А.Н. Яблонский, А.В. Новиков, В.Ю. Давыдов, П.А. Юнин, М.И. Калинников, Е.В. Скороходов, З.Ф. Красильник. ФТП, 53 (10), 1395 (2019).

[18] H. Ahmad, K. Motoki, E.A. Clinton, C.M. Matthews, Z. Engel, W. Alan Doolittle. ACS Appl. Mater. Interfaces, 12, 37693 (2020).

[19] Sh.D. Burnham, G. Namkoong, K.-K. Lee, W.A. Doolittle. J. Vac. Sci. Technol. B, 25 (3), 1009 (2007).

[20] Y.-Y. Wong, E.Y. Chang, Y.-H. Wu, M.K. Hudait, T.-H. Yang, J.-R. Chang, J.-T. Ku, W.-Ch. Chou, Ch.-Y. Chen, J.-Sh. Maa, Y.-Ch. Lin. Thin Sol. Films, 519, 6208 (2011).

[21] M. Moseley, B. Gunning, J. Greenlee. J. Appl. Phys., 112, 014909 (2012).

[22] M. Moseley, J. Lowder, D. Billingsley, W. Alan Doolittle. Appl. Phys. Lett., 97, 191902 (2010).

[23] K. Hestroffer, F. Wu, H. Li, C. Lund, S. Keller, J. Speck, U.K. Mishra. Semicond. Sci. Technol., 30, 105015 (2015).

[24] Q.M. Fu, T. Peng, C. Liu. J. Cryst. Growth, 311, 3553 (2009).
[25] D.V. Nechaev, P.A. Aseev, V.N. Jmerik, P.N. Brunkov, Y.V. Kuznetsova, A.A. Sitnikova, V.V. Ratnikov, S.V. Ivanov. J. Cryst. Growth, 378, 319 (2013).

[26] H. Hirayamaa, T. Yatabe, N. Noguchi, T. Ohashi, N. Kamata. Appl. Phys. Lett., 91, 071901 (2007).

[27] J. Bai, M. Dudley, W.H. Sun, H.M. Wang, M. Asif Khan. Appl. Phys. Lett., 88, 051903 (2006).

[28] L.F.J. Piper, T.D. Veal, C.F. McConville, L. Hai, W.J. Schaff. Appl. Phys. Lett., 88, 252109 (2006).

[29] V. Lebedev, V. Cimalla, T. Baumann, O. Ambacher, F.M. Morales, J.G. Lozano, D. Gonzalez. J. Appl. Phys., 100, 094903 (2006).

[30] X. Wang, S.-B. Che, Y. Ishitani, A. Yoshikawa. Appl. Phys. Lett., 90, 151901 (2007).

[31] Ch.S. Gallinat, G. Koblmüller, J.S. Speck. Appl. Phys. Lett., 95, 022103 (2009).

[32] V.Yu. Davydov, A.A. Klochikhin, V.V. Emtsev, D.A. Kurdyukov, S.V. Ivanov, V.A. Vekshin, F. Bechstedt, J. Furthmuller, J. Aderhold, J. Graul, A.V. Mudryi, H. Harina, A. Hashimoto, A. Yamamoto, E.E. Haller. Phys. Status Solidi B, 234 (3), 787 (2002).

[33] A.A. Klochikhin, V.Yu. Davydov, V.V. Emtsev, A.V. Sakharov, V.A. Kapitonov, B.A. Andreev, H. Lu, W.J. Schaff. Phys. Rev. B, 71, 195207 (2005).

[34] A. McAllister, D. Bayerl, E. Kioupakis. Appl. Phys. Lett., 112, 251108 (2018).

Редактор А.Н. Смирнов

\section{Features of the structural and optical properties of InGaN layers obtained by the MBE PA method with a pulsed supply of metal flows}

\section{B.A. Andreev' ${ }^{1}$, D.N. Lobanov'1, L.V. Krasil'nikova', K.E. Kudryavtsev ${ }^{1}$, A.V. Novikov' ${ }^{1}$, P.A. Yunin ${ }^{1}$, M.A. Kalinnikov' ${ }^{1}$, E.V. Skorohodov' ${ }^{1}$, M.V. Shaleev', Z.F. Krasil'nik ${ }^{1,2}$ \\ ${ }^{1}$ Institute for Physics of Microstructures, \\ Russian Academy of Sciences, \\ 603950 Nizhny Novgorod, Russia \\ ${ }^{2}$ Lobachevsky State University of Nizhny Novgorod 603950 Nizhny Novgorod, Russia}

\begin{abstract}
This paper presents the results of studying the properties of InGaN layers with a high InN content $(80-90 \%)$ obtained by molecular beam epitaxy with plasma activation of nitrogen on sapphire substrates with AlN/GaN buffer layers. The InGaN layers were formed using the metal modulated epitaxy (MME) method, as well as in nitrogen and metal rich conditions. It was found that the use of the MME method leads to a decrease in the density of threading dislocations in the InGaN layers. Nevertheless, despite the higher dislocation density, the smallest threshold of stimulated emission of $\sim 20 \mathrm{~kW} / \mathrm{cm}^{2}$ at $77 \mathrm{~K}$ was obtained for the $\mathrm{In}_{0.8} \mathrm{Ga}_{0.2} \mathrm{~N}$ layer grown under nitrogen rich conditions, which is associated with the lowest background electron concentration in this sample $\left(1.6 \cdot 10^{19} \mathrm{~cm}^{-3}\right)$.
\end{abstract}

